\title{
CODE-SWITCHING IN DER GESCHRIEBENEN SPRACHE. MEHRSPRACHIGKEIT AM BeISPIEL DEUTSCHER PRESSETEXTe, LiederTeXTE UND SMS-NACHRICHTEN UNTER BERÜCKSICHTIGUNG DER TENDENZ ZUR KONZEPTIONELLEN MÜNDLICHKEIT BZW. SCHRIFTLICHKEIT
}

\author{
Magdalena Malechová \\ Jihočeská univerzita v Českých Budějovicích, Filozofická fakulta, \\ Ústav česko-německých areálových studií a germanistiky, \\ Branišovská 31a, 37005 České Budějovice, Česká republika \\ e-mail: malechova@ff.jcu.cz
}

\begin{abstract}
Mehrsprachigkeit spielt in der heutigen Welt eine immense Rolle, ohne dass man es groß wahrnimmt. Die sprachliche Verflechtung kann bewusst oder unbewusst vorkommen, im Vordergrund steht jedoch das Verständnis der sprachlichen Interaktion. Der Beitrag zeigt einige Möglichkeiten des Aufeinandertreffens verschiedener Sprachen und die Konsequenzen eines solchen Kontaktes. Einer der Prozesse, in dem die Sprachen verschmelzen, ist CodeSwitching, und dieser Beitrag zeigt an konkreten Beispielen die in der geschriebenen Sprache koexistierenden Elemente der gesprochenen Sprache im Rahmen dieses Phänomens der Gegenwart. Das Ziel des Beitrags ist jedoch, die geschriebene Sprache zu beobachten, ihre Neigung zur konzeptionellen Mündlichkeit bzw. Schriftlichkeit zu präsentieren und an drei Textsorten zu zeigen, wie der Sprachwechsel in diesem kommunikationsausgerichteten Bereich funktioniert. Aufgrund der theoretischen Vermittlung der existierenden Formen des Sprachwechsels werden im empirischen Teil die gefundenen Code-Switching-Auszüge einer qualitativen Analyse unterzogen und die sich daraus ergebenden Schlussfolgerungen dargelegt.
\end{abstract}

\section{Keywords}

Code-switching; Conceptual verbal and written language form; Interaction; Language contact; Polylingualism.

\section{Einleitung}

Sprachkontakt stellt in der Welt eines der Grundprinzipien der zwischenmenschlichen Berührung dar. Die Sprache als Verständigungs- und Gedankenvermittlungsträger unterliegt schon immer einer ständigen Entwicklung. Mit der zunehmenden Durchlässigkeit verschiedener Landesgrenzen werden auch Sprachgrenzen als Barrieren vermindert, und die Menschen genießen einen immer freieren Kontakt auch auf dem Gebiet der Sprache. Jedenfalls führt die Durchlässigkeit der Grenzen zu einer größeren Vermischung der Sprachen durch höhere Interaktion verschiedener Nationalitäten und Sprachgruppen. Man spricht also heutzutage von einer multikulturellen Gesellschaft, die mehr und mehr an Bedeutung gewinnt. Damit einher geht natürlich die gegenseitige Beeinflussung der einzelnen Sprachen und selbstverständlich die Einflussnahme vor allem der starken Sprachen auf die weniger starken (auf der globalen Ebene könnte man als starke Sprache z. B. Englisch betrachten, auf der Landesebene z. B. in der Schweiz dominiert das Deutsche gegenüber dem Rätoromanischen). 


\section{$1 \quad$ Methodologie der Vorgehensweise}

Mehrsprachigkeit ist folglich allmählich zu einer natürlichen Tendenz geworden und gilt unter der Logik der Globalisierung als selbstverständlicher Bestandteil der gesellschaftlichen Entwicklung. Der vorliegende Beitrag handelt von einer Untersuchung der Mehrsprachigkeit in der geschriebenen Form der Gemeinsprache, wo es logischerweise viele Elemente der gesprochenen Kommunikation gibt. In dieser Studie sollen unterschiedliche schriftsprachliche Kontexte, in denen es zu einem Sprachwechsel kommt, genauer untersucht werden. $\mathrm{Zu}$ Beginn wird auf die Begriffe Mehrsprachigkeit, Code-Switching und ihre Abgrenzung eingegangen, wobei auch auf die Forschung im Bereich dieser Problematik Bezug genommen wird. Anschließend werden mögliche Perspektiven der Sprachwechselbeobachtungen umrissen, wobei die Frage der Mündlichkeit und Schriftlichkeit berücksichtigt wird. Um möglichst präzise zeigen zu können, wie sich Code-Switching in den geschriebenen Texten auswirkt, wurden drei im Rahmen der geschriebenen Sprache häufig verwendete Textsorten ausgewählt, aufgrund deren Analyse das Phänomen der Mehrsprachigkeit festzustellen ist. In diesem Zusammenhang wird die Zeitungssprache, weiter die Sprache der Lieder und die der Kurznachrichten bzw. die SMS-Kommunikation behandelt. Alle drei Textsorten haben jeweils unterschiedliche Ansatzpunkte bezüglich des Mediums bzw. des Zustandekommens, aber auch verschiedene thematische Bereiche der eigentlichen sprachlichen Anwendung. Trotzdem sind alle drei für diese soziolinguistische Untersuchung ausgewählten Quellen reichlich mit Code-Switching versehen, wie in der folgenden praktischen Analyse zu sehen ist. Da es sich bei der Untersuchung um heterogene Texte handelt, wird die jeweilige methodische Verfahrensweise unten im Einzelnen noch präzisiert.

\section{Das Forschungsziel}

In der vorliegenden Studie wird auf den Zusammenhang der linguistischen Merkmale in mehrsprachiger Kommunikation und der Motivation der einzelnen Code-Switching-Fälle eingegangen. Nach der Darstellung des anhand von drei Textsorten geschaffenen Korpus ergibt sich die Fragestellung, ob es möglich ist, in der Tendenz zur Verwendung fremder Lexik gemeinsame Aspekte bei den drei verschiedenen Textsorten zu finden, ob einzelne Textsorten artenspezifische Eigenheiten und Attribute aufweisen, und wie diese linguistisch, soziolinguistisch und funktional zu erfassen sind. Weiterhin soll untersucht werden, inwieweit konkrete Kommunikationsbedingungen der genannten Korpus-Textsorten in Bezug auf das Vorkommen des Code-Switching für konzeptionelle Mündlichkeit bzw. Schriftlichkeit sprechen, um abschließend die Tendenz anzudeuten, wie die fremde Lexik an der einen oder anderen Präferenz mitwirkt.

\section{$3 \quad$ Mehrsprachigkeit}

Mehrsprachigkeit wird als Begriff mit einer offenen Semantik verstanden [1]. Es kann damit Unterschiedliches bezeichnet werden. Einerseits ist es die Fähigkeit eines Menschen, mehr als eine Sprache zu sprechen, andererseits versteht man unter diesem Begriff die Geltung oder verbreitete Anwendung mehrerer Sprachen in einer Gesellschaft, einem Sprachgebiet oder einem Staat. Duden online fasst den Begriff Mehrsprachigkeit als „die Fähigkeit, mehrere Sprachen zu sprechen“" zusammen. [2]

Als Synonym zum Begriff Mehrsprachigkeit wird im Duden darüber hinaus der Begriff Polylingualismus benutzt. [3] Eine andere Bezeichnung wäre dann Multilingualismus, und im Zusammenhang mit diesem Begriff wird Zweisprachigkeit bzw. Bilingualismus verwendet.

Heutzutage ist Zwei- oder Mehrsprachigkeit schon ein natürlicher Bestandteil der zwischenmenschlichen Kommunikation, weil die heutige globalisierte Welt auf 
nebeneinander existierenden soziokulturellen Tatsachen und Existenzen beruht. Es wurden auch verschiedene Arten der Zweisprachigkeit beschrieben, viele Studien und Forschungen auf dem Gebiet des Spracherwerbs von Kindern durchgeführt, die oft auf den Ergebnissen der Sprachpyramide nach Wendlandt basieren. [4]

\section{$4 \quad$ Code-Switching}

\subsection{Der Begriff und bisherige Forschung}

Code-Switching stellt in der heutigen Zeit ein besonderes Sprachkontaktphänomen dar, obwohl es nicht neu ist. Es wird seit Mitte der 50er Jahre des 20. Jahrhunderts von verschiedenen SprachwissenschaftlerInnen erforscht, und im Kontext der gegenwärtigen Globalisierung wurde es immer mehr Gegenstand von Untersuchungen der SprachforscherInnen.

Laut Duden [5: 340] wird Code-Switching als ,das Überwechseln von einem Code in einen anderen (z. B. von der Standardsprache in die Mundart)" betrachtet. Der Begriff selbst kommt aus dem Englischen „,code“ - steht für Code und „to switch“ für wechseln. [6] Mit dem Wort „Code“ ist aus soziolinguistischer Sicht eine „durch die Zugehörigkeit zu einer bestimmten sozialen Schicht vorgegebene Weise der Verwendung von Sprache" definiert. [7] Laut Romaine [8: 111] sind mit dem Code verschiedene Sprachen, Dialekte oder Stile gemeint. In Anlehnung an Vesga [9: 108] wird der Terminus als Kommunikationssystem verstanden: „Als unscharfer Begriff kann damit jegliches Zeichen- bzw. Codierungssystem (sprachlich, nicht sprachlich, monolingual, bilingual, prosodisch, schriftlich, paralinguistisch etc.) zur Äußerung und Interpretation kommunikativer Absichten in Beziehung gebracht werden."

Zwar existiert die Sprachkontaktforschung schon mehrere Jahre; eine Einigkeit bei dem Definieren einzelner Begriffe herrscht jedoch nicht. Dies betrifft sowohl die Schreibung des Begriffs <Code-Switching> (bzw. <Code-switching>, <Codeswitching> [Dudenversion], $<$ Code Switching $>$ usw.) als auch die Begriffsbezeichnung. $\mathrm{Zu}$ den häufig benutzten Synonymen gehören dann Codewechsel, Sprachwechsel, Kodeumschaltung, Code-shifting, Code-alternation bzw. alternance langagière. [10: 102]

In Bezug auf die Gleichrangigkeit des Code-Switching-Begriffs mit den anderen erwähnten Synonymen schließt Müller [11] sich jedoch der Ansicht der Sprachwissenschaftlerin SilvaCorvalán [12] an und erwähnt die Abweichung in der Begriffsbezeichnung, die im Rahmen der Linguistik und aufgrund eines Kompetenzmangels für Sprachmischung geprägt wird. In diesem Sinne soll das Code-shifting einen Sprachwechsel darstellen, der als ein aufgrund von unzureichenden Kenntnissen entstehender Wechsel bezeichnet wird. [11: 9]

In der vorliegenden Arbeit wird der Begriff vor allem, jedoch nicht nur, nach der Haugen'schen Definition verwendet, und zwar wie folgt:

,[Code-Switching] occurs when a bilingual introduces a completely unassimilated word from another language into his speech". [13: 40]

Auf Deutsch kann man es so übersetzen, dass ein bilingualer Sprecher ein Fremdwort (also kein entlehntes Wort) in seinen Sprechakt einbaut. Die Definition weist eine ganz klare Abgrenzung des Begriffs auf, die natürlicherweise jedoch mit der Entwicklung und der Verbreitung des Code-Switching-Phänomens um weitere theoretische Erkenntnisse aus neueren sprachwissenschaftlichen Analysen erweitert wird. Grundsätzlich sind sich auch andere Linguisten wie Weinreich [14], Gumperz [15] oder Pfaff [16] über eine universale Definition einig, und diese spricht von einer wahlweisen Verwendung zweier Sprachen eines Sprechers innerhalb einer Sprechsituation: 
„Code-switching has been broadly defined as the use of more than one linguistic variety (language or dialect) by a single speaker in the course of a single conversation". [16: 344]

Ähnlich sieht es Poplack [17: 583]: Sie definiert Code-Switching als ,the alternation of two languages within a single discourse, sentence or constituent".

Mit der Zeit wurde logischerweise das Betrachtungsfeld der Mehrsprachigkeit immer breiter, und der phrasalen Formulierung sowie dem Wesen des Code-Switching wurde immer mehr Beachtung geschenkt. So kann man an der Jahrhundertwende bei einigen LinguistInnen eine erweiterte Version der Code-Switching-Definition beobachten, z. B. grenzt Bußmann [18: 106-107] den Terminus nicht nur auf zwei Sprachen oder zweisprachige Menschen ein, sondern behauptet, dass dies auch ,zwischen verschiedenen Sprachen [...] oder Varietäten eines Sprachsystems [...] bei bi/multilingualen bzw. bi/multidialektalen Sprechern innerhalb eines Gesprächs" der Fall sein kann. Einen ähnlichen Standpunkt vertritt auch Vesga [9: 99]. Diese betrachtet Code-Switching als eine vielschichtige Sprachkontakterscheinung, die im Rahmen der Mehrsprachigkeit einen breiten Raum einnimmt. Im engeren Sinne werde unter Code-Switching die Fähigkeit bilingualer Sprecher verstanden, mehr als eine Sprachvarietät zu verwenden bzw. zwischen Elementen von zwei oder mehr Varietäten zu alternieren.

Nach dem Jahre 2000 begannen immer mehr WissenschaftlerInnen sich mit dem Phänomen „Code-Switching“ auseinanderzusetzen, unter anderen Myers-Scotton [19], Onysko [20] oder Gardner-Chloros [21].

In den letzten zehn Jahren wurden auch verschiedene Werke zu diesem Thema veröffentlicht, darunter kürzere Textformen wie Sammelbandbeiträge und praxisorientierte Studentenprojektarbeiten sowie längere Untersuchungen in Form von Diplom- bzw. Dissertationsarbeiten und Monographien. Von den letzteren sind Veröffentlichungen von Munukka [22], Hänninen\&Karikoski [23], Kadamani [10], Riehl [24], Knospe [25], Müller [11] und Vesga [9] zu erwähnen. Die zuletzt Genannte definiert das Phänomen CodeSwitching als „Alternation, Verschiebung und/ oder Umwandlung von zwei oder mehreren soziokulturell bedingten Kommunikationssystemen in einer Interaktion". [9: 110]

Die Studien und Forschungen der genannten AutorInnen basieren sehr oft auf sprachpraktischen Analysen, die meistens aufgrund der Beobachtungen an Zuwanderungsbzw. bilingual aufwachsenden Kindern und Jugendlichen gemacht wurden oder die sich auf korpusbasierte Untersuchungen stützen. Daraus resultiert auch die Diversität der begrifflichen Interpretationen, die sich im Laufe der Zeit, aufgrund der neuen Erkenntnisse aus der Empirie, der Nonkonformität und Nicht-Beständigkeit der Sprache und gleichzeitig der Formenvielfalt des Code-Switching herauskristallisieren.

\subsection{Abgrenzung des Code-Switching}

Um dem Begriff Code-Switching noch näher zu kommen, ist es wichtig, ihn gegenüber anderen existierenden, vielleicht auch verwandten Begriffen abzugrenzen. In Frage kommen Bezeichnungen wie Fremdwort, Entlehnung, Ad-Hoc-Entlehnung, Diglossie, Code-Mixing, Polyphonie.

Ein Fremdwort ist ein ,aus einer fremden Sprache übernommenes od. in der übernehmenden Sprache mit Wörtern od. Wortteilen aus einer fremden Sprache gebildetes Wort [... $]^{\text {" }}[5$ : 575]. Es gibt auch sogenannte Gastwörter, das sind geläufig und allgemein vertraut gewordene Fremdwörter. [26]

Bei dem Begriff Diglossie geht es um „1. Form der Zweisprachigkeit, bei der die eine Sprachform die Standardsprache darstellt, während die andere im täglichen Gebrauch, in 
informellen Texten verwendet wird, 2. Vorkommen von zwei Sprachen in einem bestimmten Gebiet [...], 3. das Auftreten von zwei ausgebildeten Varianten der Schriftsprache in einem Land $[\ldots]^{“ *} .[27]$

Die genannte Definition beruht auf der von Ferguson stammenden Auffassung des Begriffs, der Diglossie folgendermaßen definiert:

Diglossie ist eine verhältnismäßig stabile Sprachsituation, in der es neben den ursprünglichen Dialekten der Sprache (die ein Standard oder regionale Standards umfassen können) eine stark abweichende, hochgradig kodifizierte (oft grammatisch komplexere) überlagerte Variante gibt, die das Medium eines großen und geachteten literarischen Schrifttums entweder aus einer früheren Epoche oder einer anderen Sprachgemeinschaft ist, die größtenteils durch formale Erziehung erlernt wird und für die meisten geschriebenen und formellen gesprochenen Zwecke verwendet wird, von keinem Teil der Gemeinschaft jedoch für gewöhnliche Unterhaltung benutzt wird. [28: 268]

Fishman [29] geht zwar von der Gumperz'schen Position [30] aus, dass Diglossie weder nur in mehrsprachigen Gesellschaften mit offiziell verschiedenen Sprachen noch in Gesellschaften, die Volkssprachen und klassische Varietäten verwenden, vorhanden ist, sondern auch in Gesellschaften mit verschiedenen Dialekten, Registern oder funktional differenzierten Sprachvarietäten; er versucht jedoch die Bewahrung der Diglossie sowie ihren Verfall auf nationaler oder gesellschaftlichen Ebene zu erforschen. [29: 96] Bei der Behandlung dieser Problematik hebt Löffler hervor, dass Standard und Dialekt in der Diglossie Varietäten mit scharf getrennten Registern sind, die phonologisch, morphologisch und durch andere Merkmale gekennzeichnet und kontextuell determiniert sind. Er vertritt die Auffassung, dass zwei sprachliche Varietäten in unterschiedlichen Situationen und zu genau abgegrenzten Zwecken streng getrennt und ohne Zwischenstufen eingesetzt werden. [31: 73]

Unter einer Entlehnung versteht man ein „aus einer fremden Sprache übernommenes Wort, das sich in Aussprache, Schreibweise, Flexion der übernommenen Sprache angepasst hat (z. B. Mauer aus lat. murus)“. [5: 1004] Laut Bußmann kommen Entlehnungen vor, wenn es zu Entwicklungen und daraus hervorgehenden Erfindungen kommt, für die es keine Bezeichnung gibt. [18: 193] Laut Vesga [9: 116] bezieht sich eine Entlehnung in der Regel auf die Integration von Elementen einer Sprache in eine andere. Sie betont hier besonders das Kriterium der Assimilation. Da die übernommenen Elemente jedoch auf verschiedenen Ebenen ins System der Empfängersprache integriert werden, sei daher die Festlegung des Assimilationsgrades oder die Determinierung der strukturellen Merkmale der Empfängersprache, um als Entlehnung eingestuft zu werden, mindestens strittig.

Bei Ad-Hoc-Entlehnungen handelt es sich um das Einbetten eines Wortes bzw. Lexemes (eines fremden Elements) einer Sprache in eine grammatische Struktur einer anderen Sprache. Poplack nennt die Ad-Hoc-Entlehnungen „,nonce borrowings“ und ist der Meinung, dass diese eine Art Zwischenform zwischen dem Sprachwechsel (Code-Switching) und der Entlehnung sind. Linguistisch gesehen verhalten sie sich wie Lehnwörter (es erfolgt eine grammatische oder sogar phonologische Integration), soziologisch gesehen verhalten sie sich wie CodeSwitches [32: 200]. Allerdings wird Poplacks Theorie von Pütz [33: 276-277] kritisiert, und auch Gardner-Chloros [21: 191] ist gegen eine strikte Trennung zwischen Code-Switching und Ad-Hoc-Entlehnung, was ein Beleg dafür ist, dass unter den SprachwissenschaftlerInnen keine Einigkeit auf diesem Gebiet herrscht.

Bei Code-Mixing fehlt eine klar abgegrenzte Sprache der Interaktion. Wenn also nicht klar ist, welche der zwei Sprachen die Matrixsprache ist, spricht man von Code-Mixing. Laut Schwitalla [34: 55] hat in diesem Fall der Sprachwechsel oft keine semantische oder 
diskurssteuernde Funktion mehr. Mußmann [35] spricht bei Code-Mixing von der Vermischung der Grammatik innerhalb einer Äußerung, wobei als Code-Switching der Wechsel zwischen zwei Grammatiken in unterschiedlichen Satzteilen in einer Äußerung bezeichnet wird.

Hänninen und Karikoski [23: 30] bestehen auch aufgrund ihrer Beobachtungen der theoretischen Studien anderer SprachforscherInnen darauf, dass die Grenzen zwischen Entlehnung und Code-Switching nicht eindeutig festzulegen sind. Es gilt nämlich nicht in allen Fällen, dass ein einzelnes Wort immer eine Entlehnung und eine aus mehreren Wörtern bestehende Einheit Code-Switching ist. Folglich gibt es ebenfalls Einzelwort-CodeSwitchings, denen sogenannte phrasale Anglizismen gegenüberstehen (die als Entlehnungen kategorisiert werden), z. B. Ups and Downs, learning by doing, oder possessive Syntagmen wie loss of control. Im Rahmen der theoretischen Verankerung des Begriffs Code-Switching erläutert Vesga [9: 109] den Terminus 'Switching' als ein komplexes und damit unscharfes Netz von Mechanismen wie Alternation, Verschiebungen (Überlappungen, Einbettungen und Bewegungen zwischen Codes) und Umwandlungen (Code-Mischung). Wegen dieser offensichtlichen definitorischen Uneinheitlichkeit wird jedoch jegliche praktische Bearbeitung von sprachlichen Daten als problematisch angesehen, was der ständigen Entwicklung der Sprache und sprachlicher Prozesse zuzuschreiben ist.

\subsection{Perspektiven der Sprachwechselbeobachtung}

Im Allgemeinen muss man davon ausgehen, dass man den Sprachwechsel aus verschiedenen Perspektiven beobachten kann. Ähnlich wie man die gesprochene und geschriebene Form der Sprache einzeln behandeln kann, sind auch beide Formen beim Code-Switching zu berücksichtigen. Aus methodischen Gründen ist hier ein wichtiges Kriterium zu beachten, und zwar das des Mediums der Realisierung sprachlicher Äußerungen. Diese basieren auf der graphischen Erfassung der Texte. Die Beobachtung der Code-Switching-Fälle in der vorliegenden Korpus-Analyse geht also auf die mediale Dimension, die sich auf die Realisationsform der sprachlichen Äußerungen bezieht, zurück. Nicht unwichtig ist jedoch dabei auch die gewählte Ausdrucksweise. Dies ist auch die Problematik, mit der sich Koch und Oesterreicher beschäftigten und von denen das bekannte Nähe-Distanz-Modell, beruhend auf der Unterscheidung von Medium und Konzeption, stammt.

\begin{tabular}{|c|c|}
\hline $\begin{array}{c}\mathrm{N} \text { Ä H E } \\
\text { Kommunikationsbedingungen }\end{array}$ & $\begin{array}{r}\text { D I S T A N Z } \\
\text { Kommunikationsbedingungen } \\
\end{array}$ \\
\hline - Privatheit & - Öffentlichkeit \\
\hline - Vertrautheit & - Fremdheit \\
\hline - Emotionalität & - keine Emotionalität \\
\hline - Situations- und Handlungseinbindung .............. & $\begin{array}{l}\text {........... - Situations-und } \\
\text { Handlungsentbindung }\end{array}$ \\
\hline - physische Nähe & - physische Distanz \\
\hline - Dialogizität & - Monologizität \\
\hline - Spontaneität & - Reflektiertheit \\
\hline usw. & usw. \\
\hline
\end{tabular}

Quelle: [36]

Fig. 1: Nähe-Distanzkontinuum nach Koch/Oesterreicher

Koch und Oesterreicher, siehe in Fig. 1 [36: 12], unterscheiden also mediale Mündlichkeit und Schriftlichkeit (phonische und graphische Realisierung der Sprache) und konzeptionelle Mündlichkeit und Schriftlichkeit (Kontinuum der Kommunikationsbedingungen). Daraus kann die Erkenntnis abgeleitet werden, dass auch in der graphischen Realisierung gesprochen- 
sprachliche Merkmale auftauchen können und umgekehrt, was eine für die folgende KorpusAnalyse wesentliche Annahme bedeutet, die später auch berücksichtigt wird.

\subsubsection{Code-Switching und sein Ursprung}

Der Prozess des Code-Switching in der gesprochenen Sprache hat seinen Ursprung in der Mehrsprachigkeit. Für Mehrsprachigkeit gibt es mehrere unterschiedliche Gründe, und daher lassen sich verschiedene Typen bzw. Arten der Mehrsprachigkeit ableiten. Laut Riehl [24] gibt es erstens die individuelle Mehrsprachigkeit, die sich auf den einzelnen Sprecher bezieht, zweitens die territoriale Mehrsprachigkeit, wobei der Sprachgebrauch in mehrsprachigen Staaten oder Regionen vollzogen wird, und drittens die institutionelle Mehrsprachigkeit, bei der die Verwendung mehrerer Arbeitssprachen in Institutionen vorkommt. Andere SprachforscherInnen ordnen in diese Aufteilung auch die gesellschaftliche Mehrsprachigkeit ein, bei der ein gegenseitiges Durchdringen der Sprachgemeinschaften herrscht, wie in Überlappungsgebieten an Sprachgrenzen. [37]

Die Ursachen der Mehrsprachigkeit sind verschieden. Grundsätzlich stützen sie sich auf die politische bzw. sozioökonomische Situation des betreffenden Territoriums, weiter auf die persönliche Situation der Menschen, die bilingual aufwachsen oder Fremdsprachen für den eigenen Bedarf erwerben. In diesem Beitrag wird auf die individuelle Mehrsprachigkeit und unter diesem Aspekt existierende Formen des Code-Switching eingegangen.

\subsubsection{Code-Switching in der gesprochenen Sprache}

Was die Typisierung des Code-Switching angeht, teilt man es nach Funktionalität auf, und zwar in funktionales und nicht-funktionales Code-Switching. Beim funktionalen CodeSwitching geht es um das bewusste Einsetzen einer zweiten Sprache. Dabei handelt es sich um äußere Faktoren oder strategische Gründe. Daher wird zwischen situationellem und konversationellem Code-Switching unterschieden.

Die äußeren Einflüsse des situationellen Code-Switching können z. B. die Gesprächspartner, der Ort oder das Thema sein. Beim konversationellen Code-Switching spielen diskursivstrategische Gründe beim Sprachwechsel eine Rolle, z. B. bei der Äußerung einer persönlichen Meinung und beim Zitieren. In diesen Fällen werden der genaue Wortlaut und die Stimmlage in der verwendeten Sprache wiedergegeben.

Das nicht-funktionale Code-Switching bezieht sich dagegen auf interne Prozesse der Sprachproduktion. Der Sprachwechsel erfolgt meist ohne direkte Absicht des Sprechers, man spricht dann von einem psycholinguistisch motivierten Code-Switching. Dies kann durch sogenannte Auslösewörter (trigger-words) hervorgerufen werden. [38] Aus verständlichen Gründen wird jedoch in dieser Arbeit auf das Thema des Code-Switching in der gesprochenen Sprache selbst nicht näher eingegangen, es wird jedoch auf spezifische Aspekte verwiesen, die auf die Zusammenhänge der geschriebenen Form der Sprache mit der gesprochenen Sprache hindeuten.

\subsubsection{Code-Switching in der geschriebenen Sprache}

Einen anderen Typ des Code-Switching, auch grammatikalischer genannt [38], stellt die geschriebene Form der Sprache dar. Auch auf diesem Gebiet ist Code-Switching keine Neuigkeit. Das beweist Gardner-Chloros [21: 20] mit ihrer Erwähnung der historischen Texte - der Briefe von Cicero aus dem ersten Jahrhundert vor Christi. Andere SprachforscherInnen weisen auf gegenwärtige Medien und computervermittelte Kommunikation wie z. B. E-Mails hin. [39: 29] 
Auf jeden Fall kann sich Code-Switching in der geschriebenen Sprache anders als in der gesprochenen Sprache verhalten, und deswegen muss man die geschriebene Form des CodeSwitching anders betrachten und untersuchen. Laut Bußmann [18: 107] werden auf formaler Ebene zwei typologische Unterscheidungen getroffen: „satzinternes“ (englisch „,intrasentential“) Code-Switching und „satzexternes“ (englisch „intersentential“) CodeSwitching. Auch diese Einteilung muss jedoch nicht immer eindeutig sein. Onysko [20: 286287] zufolge gehören zu satzinternen Code-Switchings typische englische syntaktische Einheiten (phrasale Syntagmen), die in einem Satz der Matrix-Sprache vorkommen oder die ein obligatorischer Teil eines größeren deutschen syntaktischen Rahmens sind. Das bedeutet, dass der Matrix-Sprache-Satz ohne das einbezogene Code-Switching unvollständig wäre. Als natürlicher Bestandteil des satzinternen Code-Switching sind jedoch auch Einzelwort-CodeSwitchings möglich, die der mehrsprachige Sprecher aus oft ähnlichen Gründen einsetzt, wie es beim gesprochenen Code-Switching der Fall ist.

Um satzexternes Code-Switching handelt es sich, wenn ein grammatikalisch kompletter englischer Satz in einem deutschen Text steht, ohne den der Text auch verständlich wäre [20: 287-288]. Neben dem Auftreten des einen oder anderen Typs des Code-Switching erhält die Konzeption im Rahmen der graphischen Form der Realisierung eine wesentliche Bedeutung. Wie schon oben erwähnt, tauchen gesprochen-sprachliche Elemente in der Schriftsprache auf und umgekehrt. Ob eine sprachliche Äußerung mehr oder weniger zur konzeptionellen Schriftlichkeit oder Mündlichkeit tendiert, lässt sich an sprachlichen Merkmalen und Kommunikationsbedingungen feststellen. Die mündlichen Äußerungen sind oft kontextgebunden, im Gegensatz zur Schriftlichkeit, die eher als monologisch und kontextunabhängig zu bezeichnen ist. Der konzeptionell-schriftliche Sprachgebrauch ist eher gekennzeichnet durch komplexere Strukturen, ein höheres Maß an Informationsdichte, merkmalsreichere Lexik. [40: 19]

\section{$5 \quad$ Praktische Analyse der geschriebenen Form des Code-Switching}

\subsection{Presseschlagzeilen und Pressetextauszüge}

Eines der Medien, in dem das geschriebene Code-Switching vorkommt, sind Pressetexte. Auch aufgrund des Prozesses der Entstehung einer Zeitung, wo die Texte kontrolliert werden, ist die geschriebene Form des Code-Switching ganz absichtlich, weniger spontan und gar nicht unüberlegt, was bei der gesprochenen Form des Code-Switching anders ist. Trotzdem bemühen sich auch AutorInnen von Zeitungstexten um eine Art kommunikativer Unmittelbarkeit, die sie zu vermitteln versuchen, obwohl diese hauptsächlich stilistischen Zwecken dient.

Als Quelle der Beispiele für die verschiedenen Formen des Code-Switching wurden unterschiedliche Pressetexte und Presseschlagzeilen verschiedenen Zeitungen, wie z. B. die aktuelle [41], Kronen Zeitung [42], TV für mich [43] (alle Druckform), Bild-Zeitung [44], Die Welt [45] (beide online) entnommen. Das Korpus, bestehend aus Schlagzeilen und Pressetexten, wurde stichprobenweise zusammengestellt, um eine objektive Ermittlung durch die Diversität sowohl des Mediums als auch der Textform zu erzielen.

Es gibt Unterschiede zwischen dem Aufkommen des fremden Wortguts in den Schlagzeilen und in den eigentlichen Pressetexten. Die Schlagzeilen zeichnen sich durch Kürze und sehr oft durch elliptische Formen der Äußerungen aus. Deshalb ist die Grenze zwischen dem Code-Switching (CS) und einer Ad-Hoc-Entlehnung (AHE) sehr eng. Außerdem weisen vor allem die Schlagzeilen eine starke Tendenz zur Nominalisierung auf, was die Wahl des fremden Wortguts erleichtern kann. Ein paar Beispiele aus den oben genannten Zeitungen wären: 
(1) Neuer Look - CS [41: 4/2015-03-28]

(2) Stylen Sie sich jünger! - AHE [41: 4/2015-03-29]

(3) Harte Biker mit weichem Kern - AHE [42: 14/2015-03-29]

(4) Rapper Nazar punktet mit Coolness und Wortwitz - CS [42: 5/2015-03-29]

(5) Erster Weltkrieg im Comic-Look - CS [44: 2014-06-27]

Was die eigentlichen Pressetexte betrifft, wird Code-Switching nicht in jeder Zeitung und nicht immer verwendet. Das Hauptauswahlkriterium liegt dem Merkmal des funktionalsituationellen Code-Switching der gesprochenen Sprache nahe. Eine große Rolle spielt hier das Thema des Textes. Sehr wichtig sind auch örtliche Umstände, die beschrieben werden, und nicht zuletzt die konkreten Zusammenhänge der Sprache und der beschriebenen Situation, was z. B. die Vermittlung des lokalen Kolorits verschaffen kann. Die folgenden Beispiele des Code-Switching kommen aus der online-Version der Zeitung Die Welt [45].

\section{Schlagzeilenbeispiele}

(6) Reise-News - Kompositum, CS hier als eine ökonomische Variante [46]

(7) ,Don't cry“ - ein selbständiges Syntagma, kann jedoch auch als phrasaler Anglizismus betrachtet werden. [47]

(8) Let's go Europe! So billig war's dort lange nicht - satzexternes CS [48]

(9) Reisen abseits der Sightseeing-Highlights - satzinternes CS [49]

(10) Und wie hoch ist dein ,wife bonus “, Honey? - satzinternes CS mit satzexternem CS gemischt. [50]

Pressetextauszüge aus dem im online veröffentlichten Artikel mit dem Titel Und wie hoch ist dein ,wife bonus“, Honey? [50]

Satzinternes CS:

(11-12) Das Leben als ,,Wife“: Man wohnt im Penthouse auf der Park Avenue, vertreibt sich den Tag mit Core-Fusion-Kursen - und ignoriert das Elend um einen herum so gut es geht.

(13-17) Sie beschließt, die erfahrene Kränkung mit einer pseudoanthropologischen Studie zu überwinden. Herausgekommen ist „Primates of Park Avenue. A Memoir “(Simon \& Schuster), eine Abrechnung mit den sogenannten Glam SAHMs, glamourösen ,, stay-athome-moms ", ihren Spielregeln und Privilegien wie dem wife bonus zum Jahresende. Ein Werk, nach dessen Veröffentlichung sich Wednesday Martin besser nicht mehr auf der Madison Avenue zeigen sollte. Im Internet wird sie bereits als „Nestbeschmutzerin“ angeprangert, weil sie ihre girl friends verraten habe. Ein Kommentar lautet beispielsweise: „Ja, ich bekomme einen wife bonus und hab ihn verdient, also STFU. “ Letzteres steht für shut the fuck up.

In dem letzten Beispiel kommen eher phrasale Anglizismen vor, die eben auch als CodeSwitchings betrachtet werden können.

Als weitere Beispiele, diesmal für Einzelwort-Code-Switchings, können folgende Passagen aus dem oben erwähnten Presseartikel dienen:

(18-19) So bringt man die Zeit bis zum Lunch schnell rum. In High Heels trifft man sich zum gegrillten Branzino (36 Dollar) im Restaurant „Sant Ambroeus“, wo man mindestens einmal am Tag gesehen werden sollte.

(20) Nun wohnt sie auf der anderen Seite des Parks und denkt vielleicht darüber nach, ob es nicht besser gewesen wäre, die Sache mit den mean girl moms - den Zickenmütternmit weniger Sozialneid und mehr Humor anzugehen.

(21) Außerdem ist in Downtown noch viel mehr Geld. 
(22) Zu meiner Zeit kamen die Mütter ungeschminkt. Und niemand hatte eine Birkin_Bag.

Im letztgenannten Beispiel (22) bei Birkin Bag könnte man Bag als Code-Switching nur dann betrachten, wenn es sich nicht um den Bestandteil eines Markenzeichens handeln würde. Andernfalls ist Birkin Bag als eine Einheit des Markennamens anzusehen.

Bezüglich der Schilderung der Schlagzeilen- und Pressetexte-Untersuchung kann man im Rahmen der Kommunikationsbedingungen sowohl Aspekte der Schriftlichkeit als auch der Mündlichkeit beobachten. Für die Schriftlichkeit sprechen vor allem die höhere Informationsdichte, die Planung und die raumzeitliche Trennung der Sprachproduktion und Monologizität. Letztere wird jedoch oft durch dialogische und interaktive Sprechakte ersetzt, die anhand von Fragen und elliptischen Teilsätzen gebildet werden. Die Elemente der Mündlichkeit kann man an der Affektivität und der künstlich herbeigeführten Spontaneität der Aussagen identifizieren. Es wird auch oft Vertrautheit und damit zusammenhängende Privatheit anhand von direkten Ansprachen der LeserInnen geschaffen. Die Anrede der Leserschaft durch die persönliche Note in Verbindung mit Aufforderungen in Form von z. B. Ratschlägen, wie etwa in (7) „Don't cry“ bzw. in (8) Let's go Europe, zeigt die Tendenz zu einer face-to-face-Interaktion, die eigentlich ein grundsätzliches Merkmal der konzeptionellen Mündlichkeit ist.

Die Pressetexte wollen mit ihren Code-Switchings eine emotionale Atmosphäre schaffen, was vor allem mithilfe von derben Ausdrücken und Schimpfwörtern wie etwa in (17) die Abkürzung STFA oder umgekehrt mit Bezeichnungen, die einen Liebesbeweis liefern, wie z. B. Honey in (10) geschieht. Diese Passagen zeigen wiederum die Tendenz zur konzeptionellen Mündlichkeit. Es lässt sich beobachten, dass die Zeitungssprache nicht nur inhaltlich, sondern auch formal vom Aspekt der Aktualität ihrer Produktion regiert wird. Der Anglizismen-Wortbestand und vor allem der dadurch initiierte Sprachwechsel sind hier folglich ein Zeichen des „Auf-dem-neuesten-Stand-Status“ bzw. des „In-Seins“. Trotz der scheinbaren Resonanz mit den Mündlichkeitsprinzipien bleibt die Textproduktion der Zeitungssprache ein durchaus durchdachtes Verfahren, und deshalb erweist sich hier stärker die Tendenz zur konzeptionellen Schriftlichkeit.

\section{$5.2 \quad$ Songtexte}

Neben den Pressetexten existieren auch noch andere Quellen, wo man die geschriebene Form des Code-Switching antreffen kann. Eine der Quellen stellen Liedertexte bzw. Songtexte dar.

Diese repräsentieren in erster Linie die geschriebene Form der Sprache. Da die Songs aber später dem Publikum vorgesungen werden, kann man sie als Zwischenstufe der geschriebenen und der gesprochenen Sprache betrachten. In diesem Zusammenhang wird auch auf die Frage der Tendenz zur konzeptionellen Mündlichkeit bzw. Schriftlichkeit eingegangen.

Einer der bekanntesten deutsch singenden Interpreten und einer der ersten Protagonisten mehrsprachiger Texte (Deutsch, Englisch und andere Sprachen) war der österreichische Sänger, der unter dem Namen Falco aufgetreten ist. Der in den 60er Jahren des 20. Jahrhunderts geborene talentierte Sänger bedeutete eine mächtige Bereicherung der deutschen und österreichischen Rock-Pop-Szene. Der Sprachwechsel gehörte zu den typischen Merkmalen seiner Lieder, darüber hinaus wechselte er nicht nur die Sprachen, sondern auch den Stil der Aussageart (Singen und Vorsprechen der Lyrik).

Als Beispiele für mehrsprachige Songtexte eignen sich mehrere von Falcos Hits: Coming Home [51] beruht auf der Zweisprachigkeit und erinnert an den Prozess der Diglossie. 


\section{(23) Coming Home}

One year ago

Ein Jahr wie eine Ewigkeit

Aber es war Liebe auf den ersten Blick

Niemand wollte uns verstehen ...

Das Lied Out of the Dark [52] beruht eher auf satzexternem Code-Switching, weil sich einzelne syntaktische Einheiten der einzelnen Sprachen ablösen und meistens nicht voneinander abhängig sind.

\section{(24) Out of the Dark}

Out of the dark - Hörst Du die Stimme, die dir sagt

Into the light - I give up and close my eyes

Out of the dark - Hörst Du die Stimme, die dir sagt

Into the light - I give up and you waste your tears to the night

Bei gründlicherer Untersuchung des Songtextes (24) kann man feststellen, dass die einzelnen Passagen sich in den Zeilen reimen. Angesichts dessen lässt sich annehmen, dass das Einsetzen von Code-Switching bewusst geplant ist, was die Präferenz der konzeptionellen Schriftlichkeit bestätigt.

Das dritte Lied Qué pasa hombre [53] repräsentiert ebenfalls das satzexterne Code-Switching. Es wechseln jedoch drei Sprachen im Text: Deutsch, Englisch und Spanisch.

\section{(25) Qué pasa hombre}

Qué pasa hombre, hombre, ah, ah, aah

Che-che-check it out hey

- ah listen listen listen

Good evening ladies and gentlemen,

this is your Flugzeugführer speakin'

We welcome you aboard of Cash \& Flight Airways

On our flight through the night

Say: shock shock

Ich gebe nur das Motto aus:

around around the clock

Noch immer das Motto: never ever stop ...

Bei diesem Song (25) lässt sich allerdings in Zweifel ziehen, ob es sich um Code-Switching handelt, da hier drei verschiedene Sprachen herangezogen wurden und es schwer ist festzulegen, welche der Sprachen die Matrix-Sprache ist. Daher bekräftigt es die Annahme, dass sich in diesem Fall schon Code-Mixing realisiert. In diesem Sinne wird der Eindruck vermittelt, dass der Text ganz spontan entstanden ist bzw. dass der Text mindestens so wirken soll, dass er spontan entstanden ist, zumal man von einer gewissen Art von emotionaler Affektivität ausgehen kann. Diese ist hauptsächlich an den verwendeten Interjektionen und Wiederholungen abzulesen. Es wäre hier also die Hypothese der Tendenz zur konzeptionellen Mündlichkeit gut nachvollziehbar.

\subsection{SMS-Nachrichten}

Die nächste interessante Quelle mehrsprachiger Texte ist das Schweizer SMS-Korpus "sms4science.ch". [54] Dieser Zusammenstellung wurden auch alle Beispiele dieses Kapitels entnommen. Sie werden weiterhin mit dem entsprechenden Datum versehen. 
Dass eine Schweizer Institution ein wissenschaftlich ausgerichtetes Korpus erstellt hat, wo man Mehrsprachigkeit als Hauptattribut einer polylingualen Gesellschaft betrachten kann, ist äußerst positiv und für nähere Untersuchung hervorragend geeignet. Das Forschungsgebiet dieser Untersuchung ist das Aufkommen des Code-Switching in zwei- bzw. mehrsprachigen SMS-Nachrichten. Die Grundkriterien der Sprachenauswahl waren folgende:

- die Metasprache (Matrix): Deutsch

- Code-Switching-Sprache: Englisch bzw. Französisch, Italienisch, Spanisch

Es wurden von den insgesamt 1876 gefundenen Treffern die ersten 500 Exzerpte bearbeitet. Viele der Beispielsätze, d. h. Sätze, Teilsätze und Wendungen, die hier gezeigt werden, können orthographische und grammatische Fehler beinhalten, die Beispiele wurden jedoch bewusst so übernommen und näher analysiert.

Betrachtet man die zwei Grundtypologien der Unterscheidung des Code-Switching, nämlich intrasentential/ satzinternes und intersentential/ satzexternes Code-Switching, sind beide Formen des Sprachwechsels häufig vertreten. Ein paar Beispiele für intrasentential/ satzinternes Code-Switching wären:

(26) Du workaholic, ich schicke dich in therapie! [54: 2009-10-27]

(27) Hallo, meine mutter bringt die girls morgen ins reiten, [...] [54: 2009-10-28]

(28) Was machst du? Ich bin am gate und gehe gleich ins flugzeug. [54: 2009-10-28]

(29-30) Cara, wie geht's? Hast Du am Donnerstag lust und zeit entweder für businesslunch oder-dinner? [...] [54: 2009-10-28]

(31-33) Hey friend, sorry für die späte antwort... war bis am sieben am schaffen und hab danach noch an meinem bett gebaut...bin voll im arsch...mach mer morgen dafür, oder? call you am mittag. Wünsch dir noch en schönen aben...gruss da bobomann [54: 2009-1028]

(34-36) Hey Ladies werde morgen auch nicht im office sein habe ne leichte magen darm grippe! Kann jemand von euch boss unterstützen falls er was braucht? Hab ihn schon informiert! Danke und schönes Weekend! Kiß [54: 2009-10-28]

Ein Beispiel für Code-Mixing innerhalb eines Satzes wäre:

(37-38) I know .....-) dank automatischem zeitupdate beim handy ....Lg [54: 2009-10-28]

Ein anderes Beispiel steht für die Ad-Hoc-Entlehnung:

(39-40) Komm nicht walken, hab grad kurzfristig Termin gekriegt beim Doc. LG Jannine [54: 2009-10-29]

Es kommt auch vor, dass die SMS teilweise durch Ad-Hoc-Entlehnungen und teilweise durch Code-Switching gefüllt wird, z. B.:

(41-44) Hallo dalibor, sorry störe dich nur sehr ungern in deinen ferien! Habe auf meinem compi das office upgedatet und jetzt benötige ich den product key ... in deinem software-schrank habe ich leider nichts gefunden. GLg, alice [54: 2009-10-29]

Was das satzexterne (intersentential) Code-Switching angeht, sind viele Fälle vertreten. Im Unterschied zu dem satzinternen Code-Switching, wo das Code-Switching innerhalb einer Satzeinheit auftritt, geschieht der Sprachwechsel beim satzexternen Code-Switching an Satzgrenzen oder nach einem Teilsatz. Satzexterne Code-Switchings bilden also selbständige syntaktische Einheiten, die oft entweder am Anfang oder am Ende einer Aussage stehen können. Oft gehören zu diesem Typ Einzel-Code-Switchings wie Begrüßungsformeln, Abschiedswörter, Antworten u. Ä. 
Die folgenden Beispiele zeigen die vor einem deutschen Satz stehenden Code-SwitchingEinheiten:

(45) Hi Mel! Musste grade an dich denken [...] [54: 2009-10-28]

(46) Thank you! Wir haben trübes Herbstwetter und feuern den Kachelofen [...] [54: 2009-10-28]

(47) Hi santi, whattamorning! Tram, alle starrn gradaus [...] [54: 2009-10-28]

(48) Ciao bella! Gehen dieses weekend nicht [...] [54: 2009-10-28]

(49) Wow so cool!!! Bin grad auf dem weg in meine 'hängematraze'...schlaf gut! ...Zzz [54: 2009-10-28]

(50) Hi my dear! Wie verlief die OP? Daf ich dich morgen abend besuchen kommen? Biiig-huge hug! [54: 2009-10-29]

In den nächsten Beispielen sieht man hinter einem deutschen Satz stehende CodeSwitchings:

(51) Salut Sima bist Du dabei heute Abend beim Federer-Match? Lets keep contact! Bisou Josi [54: 2009-10-28]

(52) Sind jetzt gerade von Triest zurück. Tolle Stadt [...]. Have fun in Göteb. M. [54: 2009-10-28]

(53) hahaha. Tschuldiqunq schadz. [...]. Love so matsch. [54: 2009-10-28]

Als nächste Beispiele treten auch solche auf, wo ein deutscher Satz in der Mitte steht. Vor und hinter dieser Phrase stehen satzexterne Code-Switchings:

(54-55) Hello darling! Wird ganz sicher spät heute! In 10min starten wir die nächste OP, [...]Du musst nicht warten! Thousand kisses A [54: 2009-10-28]

(56-57) hola chica!!! Soll dir nori die foto-cd mitbringen, [...] greezli slu-si [54: 2009-1029]

(58-59) Hi my dear! Wie verlief die OP? Darf ich dich [...]. Biiig-huge hug [54: 2009-1029]

Da es sich um ein Schweizer Korpus handelt, gibt es in vielen der SMS-Nachrichten eine Mischung von mehreren Sprachen. Die nächsten Beispiele zeigen Code-Switchings bestehend aus mehr als nur zwei Sprachen, nämlich aus drei oder sogar vier Sprachen, die auf dem Gebiet der Schweiz als offizielle sprachliche Varietäten vorkommen können. Einige SMSNachrichten sind noch um Anglizismen bereichert:

(60-61) ciao amici, steht unser lunchdate von heute noch? Gruss j. [54: 2009-10-28]

(62) Hey rubina! Ca va? na? Gut gestartet? Bin am Freitag in basel. wollen wir einen kaffee trinken gehen? biz, lucy [54: 2009-10-28]

(63-65) Liebe cony, Eine frage, könntest du mir aus den usa ein paar beef jerkey mit scharfem bbg geschmack mitbringen? Die sind so fein! (-) hihi merci und liebs grüessli [54: 2009-10-28]

Allgemein wird die SMS-Kommunikationsform dann verwendet, wenn man schnell, sprachlich frei und offen auf eine gegebene Situation reagieren will bzw. von einem SMSGesprächspartner unmittelbar eine Reaktion braucht bzw. diese vermitteln möchte. Deshalb kann davon ausgegangen werden, dass sich im Rahmen dieser Kommunikationsform relativ viele gesprochen-sprachliche Prozesse widerspiegeln. Reinkemeyer [55: 85] unterstreicht die Tatsache, dass im Rahmen der SMS-Schreibung einerseits altbekannte Eigenschaften konzeptioneller Mündlichkeit bzw. Schriftlichkeit innovativ kombiniert werden. Andererseits stellt sie fest, dass in Anpassung an die technischen Rahmenbedingungen des Kommunikationsmediums neuartige sprachliche Merkmale entstanden seien, die konzeptionell weder als typisch mündlich noch als typisch schriftlich klassifiziert werden könnten. Sie definiert die SMS-Kommunikation als ein besonderes Zusammenspiel aus 
schriftlicher Realisierungsform und konzeptioneller Mündlichkeit. [55: 84] Naheliegend ist die Frage nach den Hintergründen und Ursachen dieser beobachteten Phänomene. Dabei spielen das Zusammenwirken von technischem Fortschritt und digitaler Entwicklung eine Rolle. Vor dieser Zeit konnte nämlich eine direkte Kommunikation zwischen zwei bzw. mehr Kommunikationspartnern nur in unmittelbarer physischer Nähe stattfinden, wobei heutzutage sprachliche Äußerungen, die medial vermittelt werden, mündliche Sprachform annehmen. Diese Tatsache wird allerdings bei allen Medien mehr oder weniger sichtbar. Reinkemeyer [55: 101] nennt es medial vermittelte mündliche Kommunikation. Daraus folgt, dass trotz der physischen Distanz eine neue Art der Nähe entsteht, und zwar eine solche, die mithilfe von neuen Technologien als Sprache im Sinne des Begriffs von Schmidt [56: 126], nämlich „vermündlichte Schriftlichkeit" zustande kommt. Kilian [57: 61] wiederum führt die Bezeichnung ,geschriebene Mündlichkeit“ ein. Die daraus folgende Konsequenz kann als ein allmähliches Verschwinden der Grenze zwischen konzeptioneller Mündlichkeit und Schriftlichkeit betrachtet werden.

Anhand der obigen Beispielsauszüge der SMS-Kommunikation lässt sich, vornehmlich bei satzinternem Code-Switching, beobachten, dass dieses dort eingesetzt wird, wo situationsbedingte Aspekte betont bzw. hervorgehoben werden sollen, wie beispielsweise lokale Kennzeichnungen, was ein Treffpunkt, ein Spielraum wie etwa gate (28), office (35) und (42) darstellen kann. Auch zeitliche bzw. auf die Zeit hinweisende Markierungen werden in Form von Code-Switchings lokalisiert, wie etwa business-lunch (29), -dinner (30) und lunchdate (61). Oft werden Anglizismen für sozialbedingte Markierungen verwendet, wie etwa workaholic (26), girls (27), friends (31), Ladies (34), boss (36) oder Doc. (40). Die Semantik der gewechselten bzw. ersetzten Lexik wird dadurch nicht grundverschieden, man zielt eher auf die Emotionen des Kommunikationspartners, so dass sich dadurch auch Empfindungen oder sogar versteckte, in der realen face-to-face-Kommunikation erlebte, nonverbale Kommunikationsmittel realisieren lassen. Als Beispiel sei hier die Wahl des Anglizismus boss genannt: der Ausdruck klingt etwas abwertender und assoziiert eine starke Autorität im Unterschied zu dem deutschen Leiter, Führer oder Chef. Ladies wiederum liegt im Gegensatz zu Damen ggf. Frauen im Rahmen der stilistischen Nuancierung höher.

Die satzexternen Code-Switchings stellen den Versuch dar, der SMS-Kommunikation durch die fremdsprachlichen Gruß- und Abschiedsformeln eine sehr private Note zu geben. Da sie meistens entweder am Anfang oder am Ende der Nachricht stehen, ist die Absicht zu erkennen, eine vielleicht auch in der Realität herrschende Vertrautheit herzustellen. Am Anfang stehende fremdsprachliche Grußformeln verleihen der Nachricht die gefühlte Privatheit, und gleichzeitig sind die gewählten Ausdrücke meistens auch kurz und bündig, was die Tendenz zur Sprachökonomie bekräftigt. Sehr häufig erscheinen englische Grußformeln: Hi in (46), (47), (50), (58), Hey in (62), (31), Hello in (54), das italienische Ciao in (48), (60), das französische Salut in (51) oder das spanische Hola in (56). Auch die angesprochenen Personen werden oft statt mit ihren Namen mit Gemeinbezeichnungen für Günstlinge angeredet, wie etwa: (Hi) my dear in (50), (58), (Hello) darling in (54), (Hey) Ladies in (34), (Hey) friend in (34), (Ciao) bella in (48), (Ciao) amici in (60), (Hola) chica in (56). In den Abschiedsformeln treten phraseologische Syntagmen auf, wie z. B.: Lets keep contact in (51), Have fun [...] in (52), Biiig-huge hug in (50). Alle diese fremdsprachlichen Code-Switchings sollen die Mündlichkeit im Rahmen der Schriftlichkeit übertragen und die wirkliche physische Distanz verringern. Fast alle SMS-Nachrichten zeichnen sich durch ihre emotionale und damit auch expressive Art der Darstellung aus. Koch und Oesterreicher fassen die expressive Mündlichkeit einerseits als Innovationskraft des Sprachwandels auf und andererseits als Charakteristikum der starken emotionalen Beteiligung in der Nähesprache. 
Dabei äußern sie sich auch zum Vorkommen der Emotionen in konzeptioneller Schriftlichkeit:

\section{„[...] Wir wollen damit natürlich nicht unterstellen, daß Formen der emotionalen Beteiligung nicht auch in konzeptioneller Schriftlichkeit vorkommen. “ [58: 68]}

\section{Fazit}

Es lässt sich beobachten, dass heutzutage viele verschiedene Formen des Sprachkontaktes üblich sind. Dies betrifft sowohl die mündliche Kommunikation als auch die schriftliche Form des sprachlichen Kontaktes. Beide Kommunikationsformen weisen ihre Positiva auf, aber man will sich vor allem auf ungehinderte Art und Weise und gleichzeitig möglichst schnell verständigen. Der Code-Wechsel bzw. Sprachwechsel wird dabei keineswegs als Hindernis angesehen. Im Gegenteil. Basierend auf der qualitativen Auswertung geht aus der obigen Analyse hervor, dass Code-Switching in der schriftlichen Form der Sprache divergente Medien betreffen kann. Hier wurde das Vorkommen des Code-Switching an drei unterschiedlichen Textsorten aus drei verschiedenen Medien dargestellt, nämlich an den Schlagzeilen und Pressetexten im Rahmen der Zeitungssprache, an den Songtexten und den SMS-Nachrichten.

Code-Switching ist ein unabdingbarer Bestandteil der Zeitungssprache. JournalistInnen verwenden den Sprachwechsel ganz bewusst und aus guten Gründen. Im Hinblick auf die allgemeinen Gesetzmäßigkeiten, unter denen sich die Zeitungssprache realisiert, liefern die aus der Analyse hervorgehenden Ergebnisse die Erkenntnis, dass die Tendenz zur Kürze das Code-Switching-Vorkommen nicht ausschließt, sondern eher begünstigt, denn fremdsprachliche Code-Switchings sind oft kürzer von der Form her, bieten eine Menge Nomen, die effektiv zusammengesetzt werden, so dass eine schlagzeilenförmige nominale Phrase entsteht, die sich auch noch durch die fremde Lexik auf die Emotionen der Leserschaft auswirkt. Das Appellieren an die emotionale Seite der Leserschaft durch die vertraute DuAnrede verschafft den Schlagzeilen einen vorwiegend privaten Ton, wie man ihn in der mündlichen Kommunikation pflegt. Da jedoch die Zeitungssprache vorher gut geplant und organisiert ist, weist sie in Bezug auf die Kommunikationsbedingungen sprachlicher Äußerung vorwiegend Merkmale konzeptioneller Schriftlichkeit auf. Die Merkmale der Mündlichkeit, wie etwa Vertrautheit der Partner, Kooperation, emotionale Beteiligung oder Spontaneität, werden in die Schriftlichkeit der Zeitungssprache eingeführt, sind jedoch bis ins Detail durchdacht und deswegen als gespielt und unecht zu bezeichnen.

Die Sprache der Songtexte stellt eine spezifische mediale Varietät dar. Die Songtexte werden zunächst schriftlich fixiert, und dieser Prozess kann jeweils eine gut durchdachte Handlung verkörpern oder wiederum eine sehr spontane Flut von Gedanken bedeuten, die gleich verzeichnet werden. In der zweiten Phase werden dann die verschriftlichten Texte mündlich dargeboten, meistens gesungen. Die untersuchten Songs weisen durch das Vorkommen von Code-Switchings jedoch Merkmale sowohl konzeptioneller Schriftlichkeit als auch Mündlichkeit auf. Für die Mündlichkeit sprechen die geringere sprachliche Komplexität der Textproduktion, unvollständige Teilsätze, Gedankenbrüche und der Wechsel mehrerer Fremdsprachen. Man kann auch an dieser Textsorte gut beobachten, dass es oft von einer individuellen Betrachtung der sprachlichen Äußerung abhängt, ob der Text als mehr oder weniger schriftlich bzw. mündlich $\mathrm{zu}$ bestimmen ist, zumal viele Kommunikationsbedingungen den LeserInnen bzw. HörerInnen vorenthalten bleiben können, wie z. B. der oben erwähnte Prozess der Entstehung eines Songs.

Mit Hilfe des Code-Switching wird darüber hinaus sehr oft in der inoffiziellen Sprache kommuniziert, was an Beispielen aus dem Schweizer SMS-Korpus präsentiert wurde. Die 
Untersuchung illustriert somit einen regen Sprachaustausch, bei dem gezielt, jedoch aufgrund der gelebten Mehrsprachigkeit auch irgendwie spontan auf Code-Switching zurückgegriffen wird. Die SMS-Kommunikation beruht auf einem auf Sprachökonomie aufgebauten schriftlichen Kommunikationsmuster, so dass eine Menge von gesprochen-sprachlichen Elementen offensichtlich ist, z. B. elliptische Teilsätze, phraseologische Syntagmen, offene syntaktische Strukturen, Akronyme und die bereits erwähnten Code-Switchings. Im Rahmen des von Reinkemeyer eingeführten Begriffs „langage SMS“, der als SMS-spezifischer Schriftcode bezeichnet wird, gibt es nämlich verschiedene Verschriftungsstrategien dieser Kommunikationsart. Reinkemeyer [55: 112, 121] nennt dies „Verschriftungsstil“, d. h. die jeweilige graphische Repräsentation der phonisch realisierbaren Äußerung. Festzuhalten ist, dass eine SMS-Kommunikation prinzipiell zur konzeptionellen Schriftlichkeit neigt, obwohl die Grenzen der Kommunikationsbedingungen im Rahmen der Nähe-Distanzunterscheidung auch verwischt werden können.

Die praktische Korpusanalyse sollte verdeutlichen, dass drei unterschiedliche, drei verschiedenen Medien entnommene Textsorten jeweils Gemeinsamkeiten bzw. Unterschiede in der Bestimmung der konzeptionellen Mündlichkeit bzw. Schriftlichkeit unter Einbeziehung des Code-Switching-Phänomens ihre eigenen Präferenzen aufweisen. Diese beruhen, von der jeweiligen medialen Grundlage ausgehend, auf zu unterscheidender Betrachtungsweise. Alle drei Korpus-Einheiten beinhalten Texte, die als medial-funktionale Varietäten zu bezeichnen sind. Medien allgemein zeigen typische, auch voneinander abweichende Charakteristiken, aufgrund deren dann die Tendenzen der Versprachlichung beobachtet werden können. Während Schlagzeilen, Pressetexte, aber auch Songtexte tendenziell der Seite der Schriftlichkeit zuzuordnen sind, weisen die SMS-Nachrichten vor allem aufgrund ihrer kommunikativen und dialogischen Strukturen die Tendenz zur konzeptionellen Mündlichkeit auf. Davon zeugt vorwiegend die Neigung zu eingeschränkter Syntax, Reduktionen, Abkürzungen, Akronymen. Die gelebte Mehrsprachigkeit und das spontane Einsetzen von Code-Switching spielen hier eine nicht unwichtige Rolle. Obwohl jede Textsorte eigene Strategien für die Verwendung von Code-Switching nutzt und aufgrund der mehr oder weniger mitwirkenden Kommunikationsbedingungen eigene Präferenzen der konzeptionellen Schriftlichkeit bzw. Mündlichkeit aufweist, kann hier von einer hybriden Sprachvarietät ausgegangen werden, deren Mündlichkeit- und Schriftlichkeit-Grenze tendenziell verschmilzt. Die Idee der Mischung von Mündlichkeit und Schriftlichkeit bekräftigt so die Auffassung, dass mediale Varietäten wie etwa Zeitungssprache, die SMS-Kommunikation oder auch die Songtexte in gewisser Weise hier als hybrid qualifiziert werden können.

\section{„Prinzipiell gilt sogar, daß alle Äußerungsformen, gleich welcher Konzeption, aus der für sie typischen Realisierung in das jeweils andere Medium „transferiert" werden können. “ [36: 6]}

Zusammenfassend lässt sich sagen, dass nicht nur der Sprachwechsel ein gegenwärtig blühendes sprachliches Phänomen ist, das von vielen oft und gern verwendet wird, aber auch von Sprachpuristen abgelehnt werden kann, sondern auch der Prozess des zwischenmedialen Wechsels quer durch die graphische bzw. phonische Realisierungsform möglich ist und dass den Tendenzen entweder zur Schriftlichkeit oder zur Mündlichkeit sprachliche Kommunikationsbedingungen zugrunde liegen.

\section{Literatur}

[1] Mehrsprachigkeit. [online]. [Abrufdatum 2020-04-11] Verfügbar unter WWW: https://www.fuen.org/de/fileadmin/user_upload/Dokumente/Dokumente-3_Unsere$\underline{\text { Kampagne-language-diversity Sprachenvielfalt-und-Mehrsprachigkeit-in-Europa.pdf }}$ 
[2] Mehrsprachigkeit. [online]. [Abrufdatum 2019-06-07]. Verfügbar unter WWW: https://www.duden.de/suchen/dudenonline/Mehrsprachigkeit

[3] Polylingualismus. [online]. [Abrufdatum 2019-06-07]. Verfügbar unter WWW: https://www.duden.de/rechtschreibung/Polylingualismus

[4] Die Sprachpyramide nach W. Wendlandt. In Modellprojekt: Kinder-Welten entdecken, soziale und kulturelle Integration in gesellschaftlichen Brennpunkten Sachsen Anhalts. Spracherwerb im Kindergarten. pp. 47. [online]. [Abrufdatum 2020-03-30]. Verfügbar unter WWW: https://www.vhs-st.de/familienwelten/wpcontent/uploads/2011/12/Erzieherbroschuere_Spracherwerb.pdf

[5] DUDEN: Deutsches Universalwörterbuch Dudenverlag: Mannheim, Leipzig, Wien, Zürich. 2003. ISBN 3-411-05505-7.

[6] Codeswitching. [online]. [Abrufdatum 2018-04-18]. Verfügbar unter WWW: https://www.duden.de/rechtschreibung/Codeswitching

[7] Code. [online]. [Abrufdatum 2019-04-12]. Verfügbar unter WWW: https://www.duden.de/rechtschreibung/Code\#b2-Bedeutung-3

[8] ROMAINE, S.: Bilingualism. Oxford: Basil Blackwell, 1989, pp. 337. DOI: $\underline{10.1017 / S 0047404500014858}$

[9] VESGA, D.: Code-Switching in multiperspektivischer Betrachtung. Thomas Martin Verlagsgesellschaft, München: AVM, 2018. ISBN 978-3-96135-005-6.

[10] KADAMANI, M.: Eine sprachpraktische Analyse zur Beurteilung der Schreibkompetenz arabischer Grundschulkinder im Deutschen: Inwiefern wirkt sich das Sprachwahlverhalten der Eltern auf die Schriftsprache der Kinder im Deutschen aus? [online]. 2013. [Abrufdatum 2019-06-07]. Verfügbar unter WWW: https://depositonce.tu-berlin.de/bitstream/11303/3865/1/Dokument_41.pdf

[11] MÜLLER, N.: Code-Switching. 7 wichtige Punkte für einen erfolgreichen Start ins Thema. Narr Francke Attempto Verlag GmbH + Co. KG, Tübingen, 2017. ISBN 9783823380887.

[12] SILVA-CORVALÁN, C.: Code-Shifting patterns in Chicano Spanish. In Elías-Olivares L. (Hg.): Spanish in the U. S. Setting. Beyond the Southwest. VA: National Clearing House for Bilingual Education, Rosslyn, 1983, pp. 69-88. ISBN 0-89763-073-4.

[13] HAUGEN, E.: Bilingualism in the Americas. A bibliography and Research Guide. University of Alabama Press, Montgomery, 1956.

[14] WEINREICH, U.: Languages in contact, findings and problems. New York: Linguistic Circle of New York, New York, 1953.

[15] GUMPERZ, J. J.: Language in Social Groups: Essays by John J. Gumperz. Standford University Press. California, Standford, 1971. ISBN 978-0804707985.

[16] PFAFF, C. W.: Contacts and conflicts-perspective from code-switching research. In Pütz, M. (Hrsg.): Language Choices: Conditions, constraints, and consequences. John Benjamins Publishing Company, Amsterdam/ Philadelphia, 1997, pp. 341-361. ISBN 9789027218315. DOI: 10.1075/impact.1.24pfa

[17] POPLACK, S.: "Sometimes I'll start a sentence in Spanish Y TERMINO EN ESPAÑOL": Toward a typology of code-switching. Linguistics. 1980, Vol. 18, Issue 7/8, pp. 581-618. ISSN 0024-3949. DOI: 10.1515/ling-2013-0039 
[18] BUßMANN, H.: Lexikon der Sprachwissenschaft. Herausgegeben unter Mitarbeit von Hartmut Laufer. Stuttgart: Alfred Kröner, Stuttgart, 2008. ISBN 978-3-520-45204-7.

[19] MYERS-SCOTTON, C.: Contact Linguistics. Bilingual Encounters and Grammatical Outcomes. Oxford University Press, Oxford, 2002. ISBN 9780198299530.

[20] ONYSKO, A.: Anglicism in German: Borrowing, lexical produktivity and written codeswitching (Linguistik - Impulse und Tendenzen; 23). Walter de Gruyter, Berlin/New York, 2007. ISBN 9783110912173.

[21] GARNDER-CHLOROS, P.: Code-switching. Cambridge University Press. Cambridge, United Kingdom. DOI: 10.1017/CBO9780511609787

[22] MANUKKA, P.: Funktionen und Typen des Code-Switchings im Daf-Unterricht. [online]. 2006. [Abrufdatum 2018-08-14]. Verfügbar unter WWW: https://jyx.jyu.fi/bitstream/handle/123456789/11491/URN_NBN_fi_jyu2006602.pdf? sequence $=1$

[23] HÄNNINEN, S., KARIKOSKI, T.: Crazy, Emo und andere neue Anglizismen sowie Code-Switching aus dem Englischen in der deutschen Jugendsprache. Ihre Form und Funktion am Beispiel des Jugendmagazines POPCORN. [online]. 2011. [Abrufdatum 2019-06-14]. Verfügbar unter WWW: https://jyx.jyu.fi/bitstream/handle/123456789/25955/URN:NBN:fi:jyu201101301179.pdf? sequence $=1$

[24] RIEHL, C. M.: Sprachkontaktforschung. Eine Einführung. 3. Auflage, Narr Verlag, Tübingen, 2014. ISBN 978-3-8233-6826-7.

[25] KNOSPE, S.: Entlehnung oder Codeswitching? Sprachmischungen mit dem Englischen im deutschen Printjournalismus. Peter Lang Verlag, Frankfurt am Main, 2014. ISBN 978-3-653-05070-7.

[26] Gastwort. [online]. [Abrufdatum 2019-06-12]. Verfügbar unter WWW: https://www.duden.de/suchen/dudenonline/Gastwort

[27] Diglossie. [online]. [Abrufdatum 2019-06-07]. Verfügbar unter WWW: https://www.duden.de/rechtschreibung/Diglossie

[28] FERGUSON, Ch. A.: Diglossie. In: Steger H. (Hg.): Diglossie. Anwendungsbereiche der Soziolinguistik. Wissenschaftliche Buchgesellschaft, Darmstadt, 1982, pp. 253-276. ISBN 978-3534071838.

[29] FISHMAN, J., A.: Soziologie der Sprache. Eine interdisziplinäre sozialwissenschaftliche Betrachtung der Sprache in der Gesellschaft. Max Hueber Verlag, München, 1975. ISBN 9783190067527.

[30] GUMPERZ, J. J.: Types of Linguistic Communities. Readings in the Sociology of Language. 1968. DOI: $10.1515 / 9783110805376.460$

[31] LÖFFLER, H.: Germanistische Soziolinguistik. Erich Schmidt Verlag, Berlin, 2005. ISBN 9783503079353.

[32] POPLACK, S.; MEECHAN, M.: Patterns of language mixture: nominal structure in Wolof-French and Fongbe-French bilingual discourse. In: Muysken, P. \& Milroy, L. (Hrsg.): One Speaker, Two Languages. 1995. DOI: 10.1017/CBO9780511620867.010. [Abrufdatum 2020-02-17]. [online]. Verfügbar unter WWW: http://www.sociolinguistics.uottawa.ca/shanapoplack/pubs/articles/PoplackMeechan199 $\underline{5 . p d f}$ 
[33] PÜTZ, M.: Sprachökologie und Sprachwechsel: Die deutsch-australische Sprechgemeinschaft in Canberra. Peter Lang Verlag, Frankfurt am Main/ Berlin/ Bern/ New York/ Paris/ Wien, 1994. ISBN 3-631-47304-4.

[34] SCHWITALLA, J.: Gesprochenes Deutsch. Eine Einführung. Erich Schmidt Verlag GmbH\&Co, Berlin, 2006. ISBN 9783503122967.

[35] MUßMANN, J.: Inklusive Sprachförderung. [online]. 2012. [Abrufdatum 2019-05-27]. Verfügbar unter WWW: http://www.reinhardtverlag.de/_pdf_media/M2_Zweisprachigkeit_3752.pdf

[36] KOCH, P., OESTERREICHER, W.: Gesprochene Sprache in der Romaia: Französisch, Italienisch, Spanisch. Niemeyer, Tübingen, 1990. ISBN 9783484540316.

[37] Mehrsprachigkeit. In: Wikipedia. [Die freie Enzyklopädie online]. Wikimedia project. Powered by MediaWiki, zuletzt am 30. Januar 2020 um 13:02 bearbeitet. [Abrufdatum 2020-05-27]. Verfügbar unter

WWW: https://de.wikipedia.org/wiki/Mehrsprachigkeit\#Gesellschaftliche_Mehrsprachigkeit

[38] Code-Switching. [online]. 2020. [Abrufdatum 2020-05-27]. Verfügbar unter WWW: https://de.linkfang.org/wiki/Code-Switching

[39] HINRICHS, L.: Codeswitching on the web. English and Jamaican Creole in e-mail communication. John Benjamins Publishing Co, Amsterdam, 2006. ISBN 9789027253903.

[40] KNIFFKA, G., SIEBERT-OTT, G.: Deutsch als Zweitsprache. Lehren und Lernen. 3. Auflage, Ferdinand Schöningh GmbH \& Co. KG, Paderborn/ München, 2012. ISBN 978-3-8252-3730-1.

[41] die aktuelle. Deutsche Frauenzeitschrift. Erscheint seit 1979. FUNKE Women Group GmbH, München. 2015, Nr. 14.

[42] (Neue) Kronen Zeitung. Österreichische Tageszeitung. Erscheint seit 1900. WAZ Verlag, Essen, 2015, Nr. 19732.

[43] TV für mich. 14-tägliche deutsche Frauenzeitschrift. Erscheint seit 2008. Gong Verlag $\mathrm{GmbH}$, WAZ-Mediengruppe, Ismaning, 2015, Nr. 8.

[44] Bild-Zeitung. [online]. 2014. [Abrufdatum 2017-05-28]. Verfügbar unter WWW: https://www.bild.de/schlagzeilen-des-tages/ateaserseite/der-tag-bei-bild/ateaserseite15480098.bild.html

[45] Die Welt. [online]. 2015 [Abrufdatum 2020-06-15]. Verfügbar unter WWW: https://www.welt.de/icon/article141815003/Und-wie-hoch-ist-dein-wife-bonusHoney.html

[46] Schlagzeilenbeispiel (6). [online]. 2015. [Abrufdatum 2020-06-15]. Verfügbar unter WWW: https://Www.welt.de/print/die_welt/reise/article139401547/Reise-News.html

[47] Schlagzeilenbeispiel (7). [online]. 2015. [Abrufdatum 2020-06-15]. Verfügbar unter WWW: $\quad$ https://www.welt.de/print/welt_kompakt/kultur/article137487866/Don-tcry.html

[48] Schlagzeilenbeispiel (8). [online]. 2015. [Abrufdatum 2020-06-15]. Verfügbar unter WWW: https://www.welt.de/finanzen/article139651250/Let-s-go-Europe-So-billig-wars-dort-lange-nicht.html 
[49] Schlagzeilenbeispiel (9). [online]. 2015. [Abrufdatum 2020-06-15]. Verfügbar unter WWW: https://www.welt.de/reise/gallery136825598/Reisen-abseits-der-SightseeingHighlights.html

[50] Schlagzeilenbeispiel (10), Pressetextauszugsbeispiele (11)-(22). [online]. 2015. [Abrufdatum 2020-06-15]. Verfügbar unter WW: https://www.welt.de/icon/article141815003/Und-wie-hoch-ist-dein-wife-bonusHoney.html

[51] Coming home. [online]. 2015. [Abrufdatum 2020-06-15]. Verfügbar unter WWW: https://www.youtube.com/watch?v=mpSuU2q9yMQ

[52] Out of the dark. [online]. 2015. [Abrufdatum 2020-06-15]. Verfügbar unter WWW: https://www.youtube.com/watch?v=EO96nzs4nsI

[53] Qué pasa hombre. [online]. 2015. [Abrufdatum 2020-06-15]. Verfügbar unter WWW: https://www.musixmatch.com/lyrics/Falco/Qu\%C3\%A8-pasa-hombre

[54] SWISS NATIONAL SCIENCE FOUNDATION 2011-2014. Schweizer SMS-Korpus. SMS-Beispiele (26)-(65). [online]. [Abrufdatum 2015-05-17]. Verfügbar unter WWW: http://www.sms4science.ch/

[55] REINKEMEYER, A.: Die Formenvielfalt des langage SMS im Wechselspiel zwischen Effizienz, Expertise und Expressivität. Eine Untersuchung der innovativen Schreibweise in französischen SMS. Narr Verlag, Tübingen, 2013. ISBN 9783823367437.

[56] SCHMIDT, G.: Chat-Kommunikation im Internet - eine kommunikative Gattung? In Thimm, C. (Hrsg.): Soziales im Netz: Sprache, Beziehungen und Kommunikationskulturen im Internet. Westdeutscher Verlag, Opladen/Wiesbaden, 2000, pp. 109-130. ISBN 9783531134000. DOI: 10.1007/978-3-322-90768-4_6

[57] KILIAN. J.: T@stentöne: Geschriebene Umgangssprache in computervermittelter Kommunikation. Historisch-kritische Ergänzungen $\mathrm{zu}$ einem neuen Feld der linguistischen Forschung. In Beißwenger, M. (Hrsg.): Chat-Kommunikation: Sprache, Interaktion, Soziabilität \& Identität in synchroner computervermittelter Kommunikation. Perspektiven auf ein interdisziplinäres Forschungsfeld. Ibidem-Verlag, Stuttgart, 2001. ISBN 3-89821-147-9.

[58] KOCH, P., OESTERREICHER, W.: Sprachwandel und expressive Mündlichkeit. Language change and expressive orality. Zeitschrift für Literaturwissenschaft und Linguistik. 1996, Volume 26, pp. 64-96. ISSN 0049-8653. DOI: 10.1007/BF03396105

Mgr. Magdalena Malechová, Ph.D. 


\section{CODE-SWITCHING V PSANÉM JAZYCE. UKÁZKA MULTILINGUALISMU V NĚMECKÉM TISKU, PÍSŇOVÝCH TEXTECH A SMS ZPRÁVÁCH S OHLEDEM NA TENDENCI K ÚSTNÍ ČI PÍSEMNÉ KONCEPČNÍ FORMĚ JAZYKA}

Mnohojazyčnost je v současnosti víceméně nevědomě používaný fenomén. I vzhledem k propojování nejrůznějších jazyků zůstává nejdůležitějším faktorem komunikace vzájemné porozumění. Př́íspěvek poukazuje na možnosti setkávání různých jazyků a na důsledky těchto kontaktů. Jeden z procesů, ve kterém mohou jazyky ve svých formulacích splývat, je codeswitching. Studie na svých vybraných prríkladech dokazuje koexistenci prvků mluveného jazyka v psané formě jazyka. Cílem je tedy zkoumání psaného jazyka s ohledem na tendenci k ústní či písemné koncepční formě jazyka a fungování střídání jazyků v komunikativně zaměřeném psaném jazyce, prezentované na třech druzích textu. Na základě teoretických poznatků o existujících formách střídání jazykových kódů jsou $\mathrm{v}$ empirické části studie př́kladové excerpty podrobeny kvalitativní jazykové analýze a představeny výsledky výzkumu.

\section{Code-Switching in WritTen LANGUAge. Polylingualism on the EXAMPle OF GERMAN PRESS RELEASES, LYRICS AND SMS MESSAGES CONSIDERING THE TENDENCY TO CONCEPTUAL VERBAL OR WRITTEN LANGUAGE FoRM}

Multilingualism plays an immense role in today's world. This linguistic interweaving occurs, consciously or unconsciously, but the understanding of linguistic interaction is always on the front burner. The contribution shows some possibilities of the encounter of different languages and the consequences of these contacts. One of the processes in which the languages are merging is called code-switching. On concrete examples in written language, coexisting elements of spoken language are shown. The aim of the article is, however, to observe the written language and its tendency to either conceptual verbal or written form of language and to present three types of written texts and how code-switching in this field of communication works. Based on theoretical knowledge about existing forms of changing language codes, in the empirical part of the study, exemplary excerpts are subjected to qualitative linguistic analysis and research results are presented.

\section{CODE-SWITCHING W JĘZYKU PISANYM. PRZYKŁAD WIELOJĘZYCZNOŚCI W PRASIE NIEMIECKIEJ, TEKSTACH PIOSENEK I WIADOMOŚCIACH SMS Z UWZGLĘDNIENIEM TENDENCJI DO MÓWIONEJ LUB PISANEJ KONCEPTUALIZACJI JĘZYKOWEJ}

Wielojęzyczność to tak naprawdę w obecnych czasach nieświadomie używane zjawisko. Pomimo przenikania się najróżniejszych języków, najważniejszym elementem komunikacji pozostaje wzajemne zrozumienie. W artykule wskazano możliwości stykania się różnych języków oraz wynikające $\mathrm{z}$ takiego kontaktu konsekwencje. Jednym ze zjawisk, gdy w ramach sformułowań mogą się pojawiać wtrącenia $\mathrm{z}$ innych języków, jest code-switching. Badania oparte na wybranych przykładach dowodzą współistnienie elementów języka mówionego w pisanej formie języka. Celem jest więc zbadanie języka pisanego ze szczególnym uwzględnieniem tendencji do mówionej lub pisanej konceptualizacji językowej na przykładzie trzech rodzajów tekstów. W oparciu o wiedzę teoretyczną dotyczącą istniejących form naprzemiennego używania różnych kodów językowych, w empirycznej części badań jakościowej analizie językowej poddano przykładowe fragmenty. W zakończeniu przedstawiono wyniki badań. 